International Journal of Engineering \& Technology, 7 (1.9) (2018) 211-216
International Journal of Engineering \& Technology
WPC
Website: www.sciencepubco.com/index.php/IJET
Research Paper

\title{
Unconstrained handwriting recoganization based on neural network using connectionist temporal classification token passing algorithm
}

\author{
Pinagadi.Venkateswararao $^{1 *}$, S. Murugavalli $^{2}$ \\ ${ }^{1}$ Research Scholar, Sathyabama University, Chennai, India \\ ${ }^{2}$ Professor \& Head., Department of Computer Science and Engineering, Panimalar Engineering College, Chennai, India \\ *Corresponding author E-mail: venkypin@gmail.com
}

\begin{abstract}
Recognition of human handwriting which offers the new way to improve the computer interface with the human and this process is very much useful for documents. Keyword spotting refers the spontaneous recognition of handwritten text, letter, and scripts from historical hand written books and the procedure of recovering all instance of a known keyword from an article. With a specific end goal to choose new components this paper, propose "a repetitive neural system manually written acknowledgment framework" for watchword spotting. The watchword seeing is finished utilizing an adjustment of the connectionist temporat classification Token Passing calculation in coincidence with a repetitive neural system. The proposed watchword spotting technique for written by hand message utilizing neural system, with another adaptation of connectionist temporat classification Token Pass calculation with quick and reliable catchphrase spotting can be executed without utilizing any content line or portioning separate words.
\end{abstract}

Keywords: Connectionist Temporat Classification Token Passing Calculation; Keyword Spotting; Handwriting Acknowledgment; Intermittent Neural System $(R N N)$.

\section{Introduction}

Acknowledgment of unconstrained manually written characters is one the very pinnacle of engaging and empowering themes in programmed archive examination and preparing. As of late, Optical Character Recognition (OCR) has been significantly created in view of the predominance of Internet and media strategies. In OPTICAL CHARACTER RECOGNITION applications, written by hand character acknowledgment, particularly digit acknowledgment is dispersed with in postal mail arranging, bank check preparing, information section, and so forth. In late decades numerous scientists took a shot at this theme. Zeniths of the works identified with physically composed character affirmation are done in English, Chinese, Japanese and Arabic. Regardless, few presentation efforts have in like manner been done on Indian substance. A many stages plan for the affirmation of composed by hand Bengali character is represented in [1].

Searching word from handwritten document is not only incompetent as far as computational cost is concern but similarly provide reduced presentation. The manuscript iontrend of numerous authors should be distributed along, troublesome complications were faced. There are diverse sorts of examined archive having different styles of words, text style, estimate, foundation shade of the page that makes numerous deterrents in spotting watchword from such chronicled records. [2]

The unconstrained acknowledgment of transcribed content -, for example, letters, contents or whole books - has remained a consideration of amassed examination went for various ranges [3], [4].yet this issue has being comprehended. Especially, the unconstrained penmanship acknowledgment field has various written work styles must be appropriated with, serious challenges are experienced.

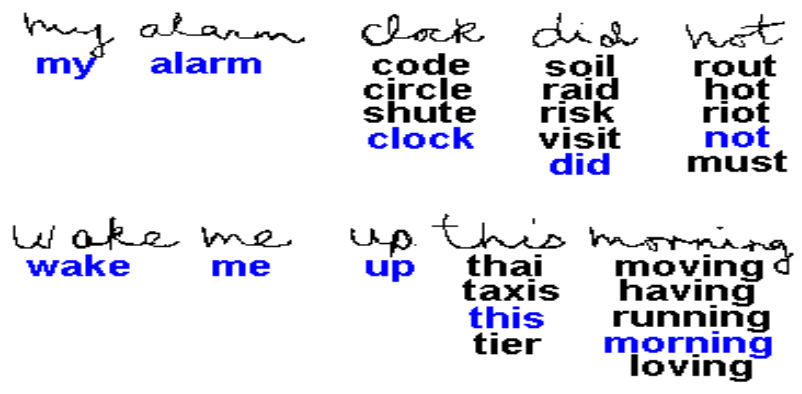

Fig. 1: Handwriting Recognition.

The photo neuron recognizes pictures Neural Signature through neural framework give a subjective vitality to instruments/PCs to believe like man cerebrum and besides the Neural imaging overhauls the speed of Image Recognition. Because of the work of neural systems the memory size and processor time have been diminished and efficient to perceive pictures through neural imaging or neural mark. [5]

Penmanship acknowledgment is routinely dispersed into on the web and disconnected acknowledgment. In online acknowledgment strategy, the 2-D directions of progressive focuses are spoken to in time space and the author strokes are additionally considered, while in the disconnected case the full report is optically checked and the finished written work is possible as a picture. In view of the more prominent simplicity of removing significant elements, online acknowledgment for the most part delivers better 
outcomes [6].due to the fleeting data accessible with the previous ,the online strategies have been appeared to be better than their disconnected partners in written by hand acknowledgment [22] [23].

On the other hand, in disconnected frameworks, the neural systems give high acknowledgment precision. In various applications tallying mail arranging, bank handling, report perusing and postal address acknowledgment include disconnected penmanship acknowledgment frameworks. As a result, the disconnected penmanship acknowledgment persists towards a dynamic territory for research to build the precision of acknowledgment [24] [25].

\section{Literature survey}

All past methodologies depend on the division of content in a picture into singular words previously continuing the catchphrase spotting. A DTW framework immediately chooses catchphrase competitors in a transcribed content line are portrayed in [8]. For general frameworks that depend on an unconstrained division, procedure is anticipated in [9] that too yield the shot of an exact division into legitimization.

The progressive idea of penmanship acknowledgment executes a few confinements on the conceivable methodologies. Straightforward semi-administered learning structures adaptable adequate to deal with the limitations forced by the many-to-few mapping of successive information are self-preparing and co-preparing. In the space of transcribed content, just couple of works has been distributed. A semi-administered adjustment to a particular author is displayed in [11], [17], where the framework is receptive to expand the acknowledgment exactness of a particular essayist be priced at lessening the unnatural acknowledgment precision. To the better information of the writers, the main effort on semiregulated studying for unconstrained penmanship acknowledgment is a previous effort finished by the writer delicate his research [10].

In [7], for single word acknowledgment, a co-preparing philosophy is utilized that shows the shrouded Markov models are more sensitive than BLSTM NN. Neural Networks (NN) has its own specific manner for catchphrase spotting through bidirectional long here and now memory (BLSTM) NN [18], [19], on the as opposed of the technique recommend in this research work. The expressed effort, contracts just with catchphrase detecting in discourse. Besides, the neural system symbolizes one watchword in the yield layer and set off the word happens in the info information. It limits the quantity of watchwords to be detected, the word must be perceived previously, and the catchphrase must happen in the preparation set.

Moreover, one hub in the yield coating of the neural system represents 1 watchword and is activated though the term happens in the info information. Along these lines, the quantity of watchwords to be detected is constrained, the term must be known previously, and the catchphrase must happen in the preparation set. A standout amongst the degree uncomplicated ways to deal with semidirected studying is by own preparing [16]. Because of its enlightening nature, it is by and large appropriate to each type of studying established order and acknowledgment framework.

It is a repetitive studying plan which hidden thought was like EM. A frail, introductoryobserver was prepared utilizing the named information as it were. Thereafter, amid the self-preparing emphases, the recognizer is utilized to mark the whole arrangement of unlabeled information and as sign a certainty measure to every characterization. The most unquestionably characterized components are thought to be marked effectively and attached to the preparation set, which is then used to prepare another observer.

The fundamental approach considers the single word pictures and receives the optical character acknowledgment (OPTICAL CHARACTER RECOGNITION). It utilizes the pixel insightful correlation to the question and the trail picture (or chose element of it, known as sectors of intrigue (ZOI)) or assesses worldwide separation esteem betwixt the 2 pixels set. Striking work in these space incorporate XOR correlation, Euclidean separation [12], Scott and Longuet-Higgins remove, Hausdorff separation of associated segments [13] also the aggregate of Euclidean separations to relating clue focuses (corner highlights) [14].

Multilayer perceptron is utilized for perceiving Manu-scripted English character [20].The highlights were removed from Borderline following and their Fourier Descriptors. Characters are recognized via examining it is structureand looking at its elements which recognize each element. It got $94 \%$ of acknowledgment with less preparing time.

Proceeding onward to the character dataset, we anticipate actualizing the managed classifier models utilized as a part of [21] and accomplish a comparative precision, and additionally fuse the strategies utilized as a part of LeCun et al. concerning shallow and profound convolution neural systems. Specifically, we can execute the convent design utilized as a part of the Lecun et al. paper, utilize exchange learning with a bigger system, and in addition execute and prepare a typical design talked about in class

\section{Research methodology}

In this paper, the watchword spotting framework depends on past work where Recurrent Neural Networks (RNN) is utilized for the penmanship acknowledgment assignment. RNN is connected to penmanship acknowledgment for proposed framework that can be classified into four procedures to be specific:

- Preprocessing,

- Feature Extraction,

- Neural network recognizer [BLSTM(Bi Directional Long Short Term Memory)]and

- Connectionist temporat classification token passing algorithm.

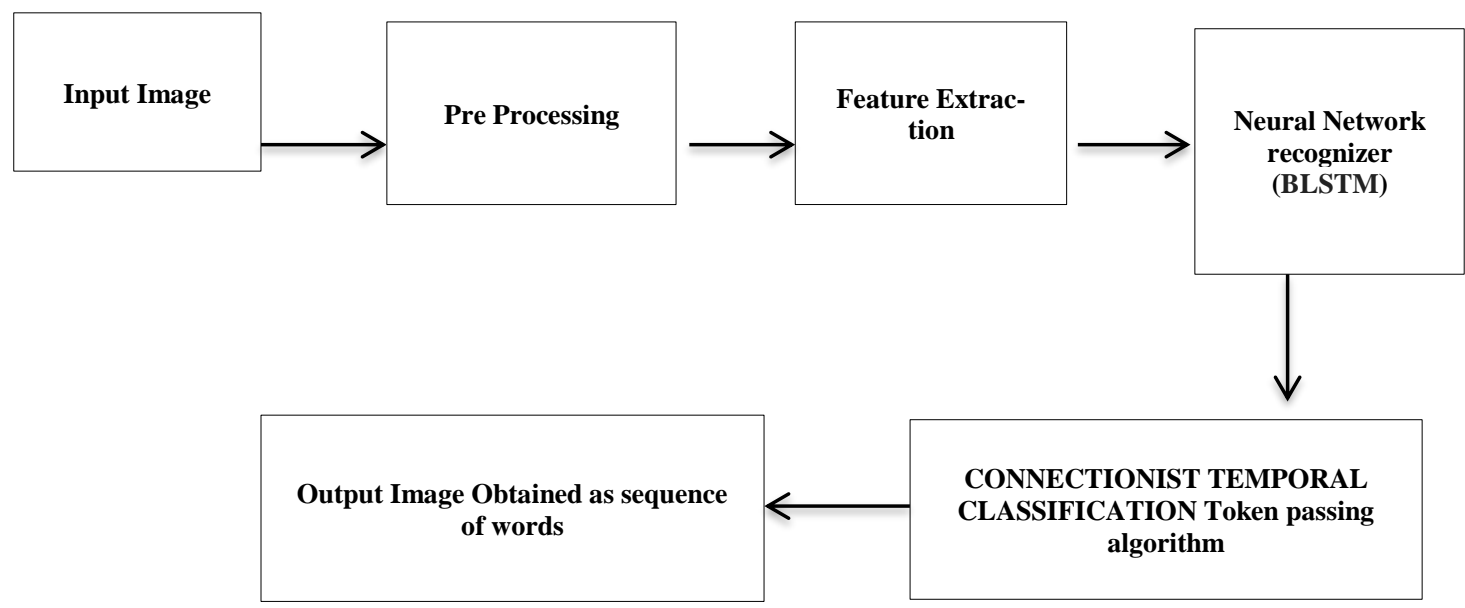

Fig. 2: Proposed Workflow Diagram. 
Preprocessing:

Pre-preparing is characterized as methodology of planning information for another procedure. The primary objectives of the preprocessing procedure areto change the data into a shape that can be all the more adequately and it plays out a progression of operations on the filtered input picture. It basically improves the picture portrayal which is suitable for portioning the information. Every individual has an alternate written work style with its own particular qualities. This reality makes the acknowledgment errand confounded. To decrease varieties in the penmanship messages, various pre-preparing operations are connected.

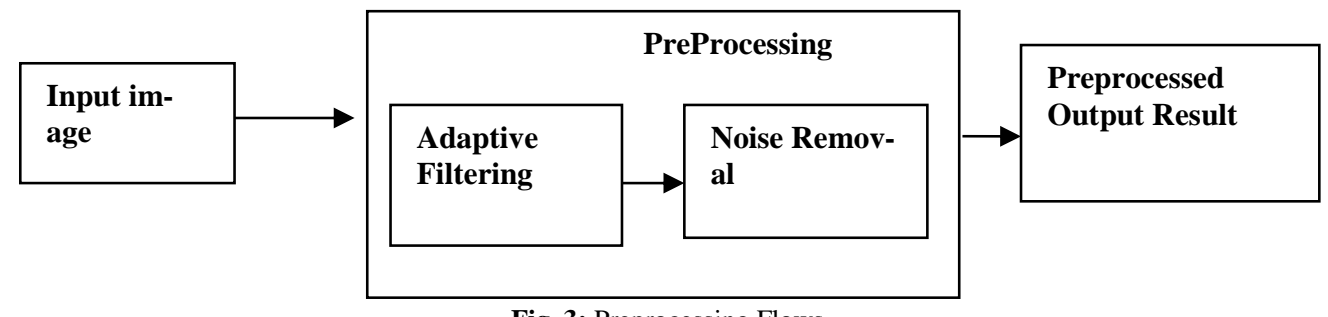

Fig. 3: Preprocessing Flows.

Adaptive filtering:

In picture handling versatile channels are for the most part used to improve or reestablish information by evacuating commotion without obscuring the picture. It is fit for modifying the clamor impact of the picture. Various versatile channels depend on the utilization of nearby pixel insights, and the change of the pixels inside the present neighborhood.

The'wiener2'function applies a Wiener channel to a picture adaptively, fitting itself to the nearby picture difference. The wiener separating is a direct strategy to limit the general mean square blunder during the time spent converse sifting and clamor smoothing. The result gives preferred outcomes over direct separating. The versatile channel is more particular than a similar direct channel, safeguarding edges and other high-recurrence parts of a picture.

A = imread ('input.jpg');

$\mathrm{X}=\operatorname{rgb} 2$ gray $(\mathrm{A})$;

$\mathrm{F}=$ imnoise (X,'gaussian', 0, 0.005);

$\mathrm{T}=$ wiener $2(\mathrm{~F},[55])$;

imshow (F)

figure, imshow (T)

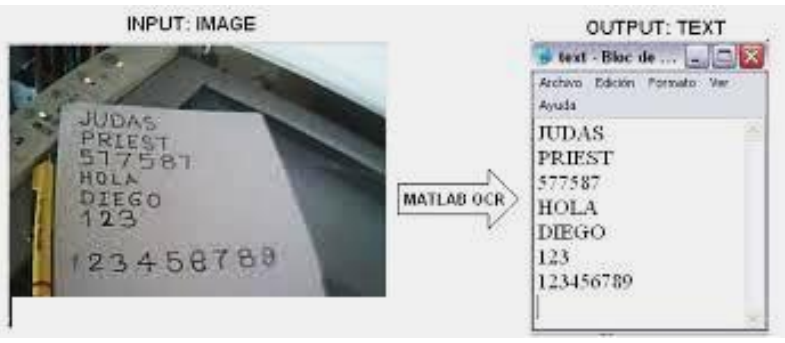

Fig. 4: Adaptive Filtering Output.

Noise Removal

Examined records regularly contain commotions that emerge because of printer, scanner, print quality, age of the archive, and so on. Pictures taken by the cameras will be loud from different sources.
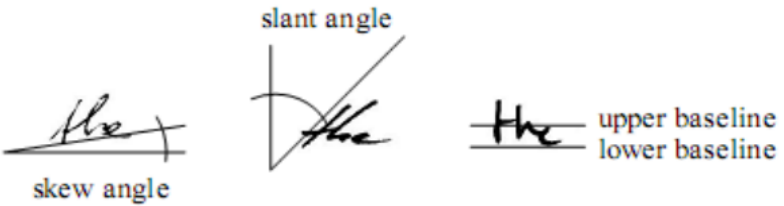

Fig. 5: Shows the Writing Characteristics of Single Person.

Feature Extraction:

The acknowledgment framework execution in view of productive elements to speak to the example attributes. The element extraction is the second stage in framework to perceive the written by hand way. Highlight extraction of the information decrease process is trailed by include investigation. Extraordinary parts of highlight examination are deciding precisely which highlights are critical.

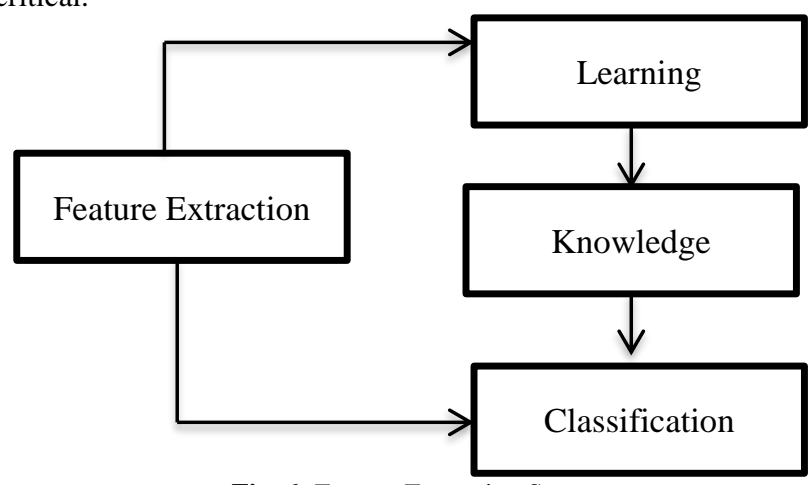

Fig. 6: Feature Extraction Steps.

From every line, a component vector is separated, that was then presented to the neural system. The element extraction is to recognize one info design from another example. It gives the qualities of the information sort to the classifier by considering the portrayal of the important properties of the picture into an element space. The reason for this progression is to remove highlights from the picture and its yields are then utilized by an example classifier. Neural network recognizer:

RNN is connectionist display with a self-associated concealed layer. One of the advantages is "memory" of prior data sources stays in the systems inner and the past setting was utilized.

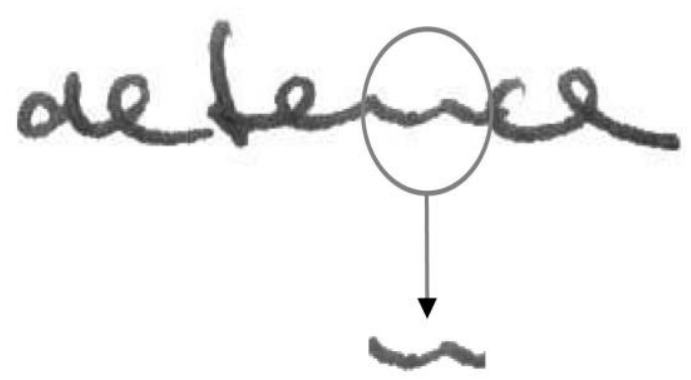

Fig. 7: Importance of Context in Handwriting Recognition.

Setting assumes an essential part in penmanship acknowledgment, as delineated in Fig. 7. "Defense" is intelligible; however the letter " $n$ " in dejection is questionable. Extra noteworthy advantage is the speed of progress of the interior states was minutely adjusted by the intermittent loads that work in vigor to confined bends of the info information.

\section{BLSTM}

The neural system portrays the succession probabilities of every character and every posture of the line. This arrangement is utilized as a part of word and perceives the word and watchword. As the Connectionist Temporat Classification token passing calculation in coincidence with BLSTM neural system. 
Connectionist Temporat Classification Token passing algorithm: The Connectionist Temporal Classification (Connectionist Temporat Classification) Token Processing calculation is fit for consolidating long-run setting in both information headings. It takes the contribution as letter likelihood succession and a dialect demonstrate, and figured.

The Connectionist Temporat Classification Token Processing figuring for single word expects a progression of letter probabilities of length $\mathrm{d}$ as yield by the framework, co-operatively with the word was a course of action of ASCII character, and returns an organizing score i.e. the probability that the commitment to the framework was beyond question the given term. Let $n(a, b)$ mean the probability of the character 1 occurring at the position $b$ as showed by the framework yield and $\mathrm{z}=\mathrm{a} 1, \mathrm{a} 2$, ln connote the word to be facilitated. The count at first broadens $w$ into a game plan.

$\mathrm{z}^{\prime}=\varepsilon \mathrm{a} 1 \varepsilon$ a2 $\varepsilon \ldots \varepsilon$ a $\mathrm{n} \varepsilon=\mathrm{v} 1 \mathrm{v} 2 \ldots \mathrm{v} 2 \mathrm{n}+1$

Additionally, makes each termvx $(x=1, \ldots, 2 n+1)$ and each posture $y=1 \ldots d$ in the accompanying line of the token $\vartheta(x, y)$ to store the probability that character $\mathrm{vx}$ is accessible at posture $\mathrm{y}$ together with the probability of the most ideal path beginning to posture y.The count steps are shown as takes after.

Require: input $\mathrm{z}=\mathrm{a}_{1}, \mathrm{a}_{2} \ldots$... an

1: Initialization:

2: expandthe one word zto another word $\mathrm{z}^{\prime}=\varepsilon \mathrm{a}_{1} \varepsilon \mathrm{a}_{2} \varepsilon \ldots \varepsilon \mathrm{a}_{\mathrm{n}} \varepsilon$

$=\mathrm{v}_{1} \mathrm{v}_{2} \ldots \mathrm{vxn}$

3: token $\vartheta(1,1)$ is equal to character $n(\varepsilon, 1)$

4: token $\vartheta(2,1)$ is equal to character $n(a, 1)$

5: Main loop:

6: for loop condition is satisfied $2 \leq \mathrm{y} \leq$ dall sequence positionsdo

7: for all positions $\mathrm{x}$ of the extended word $1 \leq \mathrm{x} \leq 2 \mathrm{n}+1$ do

8: $D_{\text {best }}=\{\vartheta(x, y-1)\}$

9: if condition $\mathrm{x}>1$ is satisfied then

10: $\mathrm{D}_{\text {best }}=\mathrm{D}_{\text {best }} \mathrm{U}$ ๆ $(\mathrm{x}-1, \mathrm{y}-1)$

11: condition if $\mathrm{v}_{\mathrm{i}}$ is not equal to $\varepsilon$ and $\mathrm{v}_{\mathrm{i}}$ is not equal tovi-2 then

12: $\mathrm{D}_{\text {best }}=\mathrm{D}_{\text {best }} \mathrm{U} \vartheta(\mathrm{x}-2, \mathrm{y}-1)$

13: condition ifend
14: condition ifend

15: token $\vartheta$ of position $(\mathrm{x}, \mathrm{y})$ is equal to $\max \left(\mathrm{D}_{\text {best }}\right) \cdot \mathrm{n}(\mathrm{x}, \mathrm{y})$

16: condition forend

17: condition forend

18: return $\max \{\vartheta(2 \mathrm{n}+1, \mathrm{t}), \vartheta(2 \mathrm{n}, \mathrm{t})\}$

For every term $\mathrm{y}$, characterize the adjusted word $\mathrm{z}^{\prime}$ as $\mathrm{z}$ with spaces included toward the start and end and between every combine of marks.

\section{Performance analysis}

The execution examination of the proposed framework is exploring the neural systems execution. All things considered catchphrase spotting accomplishes layout free detecting. i.e., it is a bit much for a catchphrase to project in the preparation sets. Utilizing Connectionist Temporat Classification calculation, the catchphrase detecting was finished with an intermittent neural system

Accuracy:

The outcomes are gotten from the system that can be ascertained by the calculation. In our work we considered manually written acknowledgment in intermittent neural system. The exactness rate is figured by deciding the quantity of accurately perceiving the obscure written by hand character from report picture.

Accuracy $=\frac{\text { number of currectly recognizing }}{\text { whole document image }}$

Error rate:

The computation of Error Rate relates input information from a transmitter and a collector. The count is finished by isolating the aggregate number of unequal sets of information components by the aggregate number of info information components from one source.

Error rate $=\frac{\text { unequal data elements }}{\text { input data elements }}$

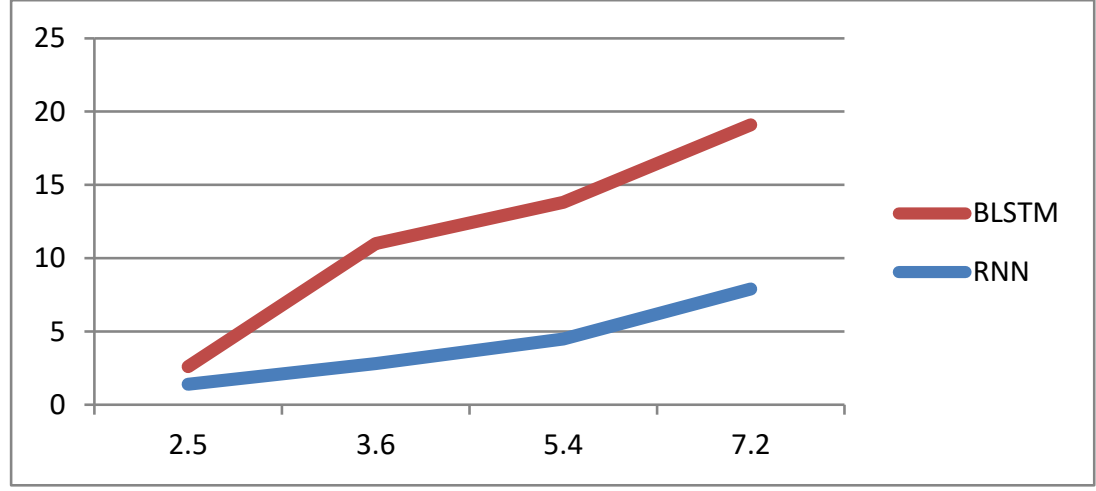

Fig. 8: Proposed Systems Accuracy Compared with Existing System

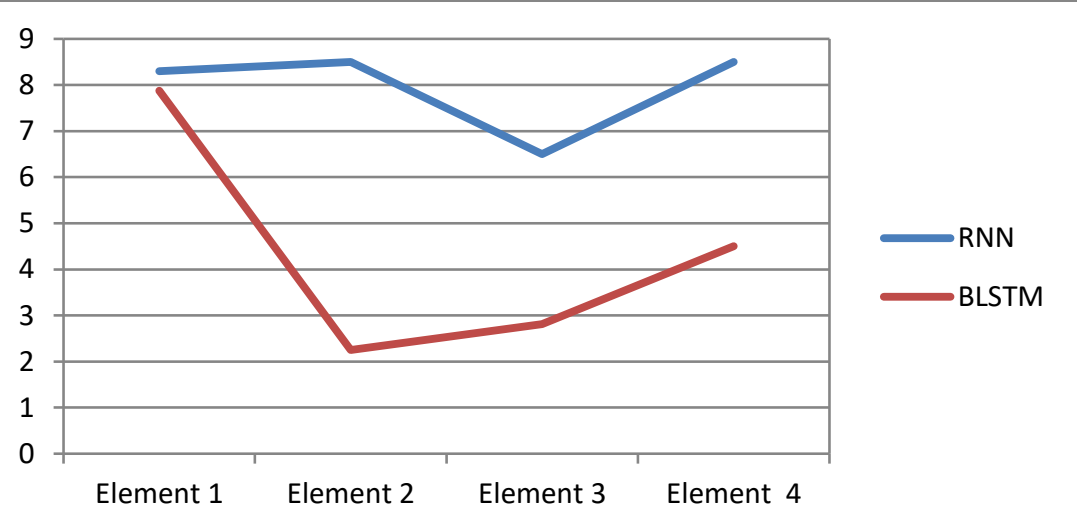

Fig. 9: Proposed Systems Error Rate Compared with Existing System. 


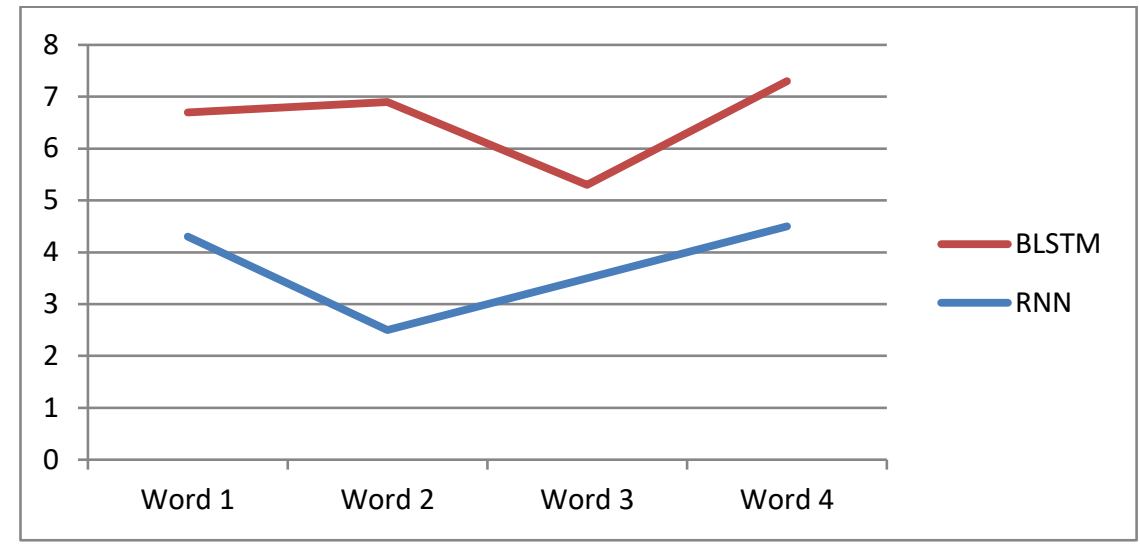

Fig. 10: Proposed Systems Recognition Rate Compared with Existing System.

Table 1: Shows the Performance Analysis of Proposed System

\begin{tabular}{lllll}
\hline & Accuracy & Time evaluation & Error rate & Recognition rate \\
\hline Artificial neural network(ANN) & $62 \%$ & $2.5 \mathrm{~ms}$ & $52 \%$ & $65 \%$ \\
Hidden & $73 \%$ & $1.9 \mathrm{~ms}$ & $45 \%$ & $71 \%$ \\
Markov model (HMM) & $81 \%$ & $1.3 \mathrm{~ms}$ & $23 \%$ & $82 \%$ \\
Recurrent Neural Network(RNN) & $93 \%$ & $0.4 \mathrm{~ms}$ & $16 \%$ & $91 \%$ \\
BLSTM(Bi Directional Long Short Term Memory) & & & \\
\hline
\end{tabular}

\section{Recognition rate:}

The acknowledgment rate is ascertained by the equation which is demonstrated as follows.

Recognition rate $=\frac{\text { number of words recognized }}{\text { number of words presented }}$

The accompanying unthinkable segment demonstrates the examination of the exactness level, mistake rate and the acknowledgment rate of the catchphrases which are given in contribution of the framework that can be performed and broke down the outcome. The outcome which is acquired from the proposed framework that can be contrasted and the current framework

\section{Conclusion}

To conclude, the penmanship acknowledgment framework sensitive to the charge of catchphrase spotting is stretchy for an assortment of writings. This paper characterized a total penmanship acknowledgment framework which has been demonstrated the mistake rate has been diminished to 23 percent and it has been demonstrated that the framework can perceive 92 percent of words. The framework execution was enhanced by permitting more casings of setting in the system inputs and the execution of neural systems was analyzed. We introduced a novel watchword spotting approach utilizing "the Connectionist Temporal Classification (Connectionist Temporat Classification) Token Passing calculation".

\section{References}

[1] Ashutosh Aggarwal, Rajneesh Rani, RenuDhir, Handwritten Devanagari Character Recognition Using Gradient Features, International Journal of Advanced Research in Computer Science and Software Engineering, Volume 2, Issue 5, May 2012, 85-90.

[2] Ms. GeetanjaliBhagwani, Ms. Ompriya Kale, "Keyword Recognition by Improving Recurrent Neural Network using Character Model", IJSRD - International Journal for Scientific Research \& Development, Vol. 3, Issue 04, 2015, ISSN (online): 2321-0613

[3] A. Vinciarelli, "A Survey on Off-Line Cursive Word Recognition," Pattern Recognition, vol. 35, no. 7, pp. 1433-1446, 2002. https://doi.org/10.1016/S0031-3203(01)00129-7.

[4] R. Plamondon and S. N. Srihari, "On-Line and Off-Line Handwriting Recognition: A Comprehensive Survey," IEEE Transaction on Pattern Analysis and Machine Intelligence, vol. 22, no. 1, pp. 6384, 2000. https://doi.org/10.1109/34.824821.

[5] Faisal Tehseen Shah, Kamran Yousaf," Handwritten Digit Recognition Using Image Processing and Neural Networks" Proceedings of the World Congress on Engineering 2007 Vol I WCE 2007, July 2 4, 2007, London, U.K.

[6] R. Seiler, M. Schenkel, and F. Eggimann, "Off-Line Cursive Handwriting Recognition Compared with On-Line Recognition," Proc. 13th Int'l Conf. Pattern Recognition, vol. 4, p. 505, 1996.

[7] V. Frinken, A. Fórnes, A. Fischer, H.Bunke, Co-training forhandwritten word recognition, in: International Conference on Document Analysis and Recognition, 2011, pp.314-318.

[8] Popescu, "A Line-Oriented Approach to Word Spotting in Handwritten Documents," Pattern Analysis and Applications, vol. 3, pp. 153-168, 2000. https://doi.org/10.1007/s100440070020.

[9] H. Cao, A. Bhardwaj, and V. Govindaraju, "A Probabilistic Methodfor Keyword Retrieval in Handwritten Document Images," Pattern Recognition, vol. 42, no. 12, pp. 3374-3382, December 2009. https://doi.org/10.1016/j.patcog.2009.02.003.

[Online].Available:

[10] V. Frinken, H. Bunke, Self-Training for hand written text line recognition, in: $15^{\text {th }}$ IberoamericanCongressonPatternRecognition, 2010, pp.104-112.

[11] G. R. Ball, S. N. Srihari, Semisupervisedlearningforhandwritingrecognition,in: 10thInternationalConferenceonDocumentAnalysisandRecognition,2 009,pp.26-30.

[12] R. Manmatha and T. M. Rath, "Indexing of Handwritten HistoricalDocuments - Recent Progress," in Symposium on Document ImageUnderstanding Technology, 2003, pp. 77-85.

[13] Y. Lu and C. L. Tan, "Word Spotting in Chinese Document Images withoutLayout Analysis," in 16th Int'l Conference on Pattern Recognition,2002, pp. 57-60.

[14] J. Rothfeder, S. Feng, and T. M. Rath, "Using Corner Feature Correspondencesto Rank Word Images by Similarity," in Workshop on DocumentImage Analysis and Retrieval, 2003, p. 30.

[15] D.Yu,B.Varadarajan,L.Deng,A.Acero,Activelearningandsemisupervisedlearning forspeechrecognition:aunifiedframeworkusingtheglobalentropyreduction maximizationcriterion,ComputerSpeechandLanguage24(3) (2010)433-444.

[16] K.Nigam,R.Ghani,Understandingthebehaviorofco-training,in:KDD2000 WorkshoponTextMining,2000,pp.105-107.

[17] P.Dreuw,D.Rybach,C.Gollan,H.Ney,Writeradaptivetrainingandwriti ng variantmodelrefinement foroffline Arabic handwritingrecognition,in:10th International ConferenceonDocumentAnalysisandRecognition,vol.1,2009, pp. 21-25.

[18] S. Fern'andez, A. Graves, and J. Schmidhuber, "An Application ofRecurrent Neural Networks to Discriminative Keyword Spotting," in17th Int'l Conf. on Artificial Neural Networks, ser. Lecture Notes inComputer Science, vol. 4669, 2007, pp. 220-229.

[19] M. Wollmer, F. Eyben, J. Keshet, A. Graves, B. Schuller, and G. Rigoll,"Robust Discriminative Keyword Spotting for Emotionally ColoredSpontaneous Speech Using Bidirectional LSTM Networks," in IEEEInt'l Conf. on Acustics, Speech and Signal Processing, 2009, pp. 3949-3952

[20] Anita Pal \& Dayashankar Singh, "Handwritten English Character Recognition Using Neural," Network International Journal of Com- 
puter Science \& Communication.vol. 1, No. 2, July-December 2010, pp. 141-144.

[21] T. E. de Campos, B. R. Babu, and M. Varma. Character recognition in natural images. In Proceedings of the International Conference on Computer Vision Theory and Applications, Lisbon, Portugal, February 2009

[22] R. Plamondon and S. N. Srihari, "On-line and off- line handwritten characterrecognition: A comprehensive survey,'IEEE. Transactions on Pattern Analysis andMachine Intelligence, vol. 22, no. 1, pp. 63 $84,2000$.

[23] N. Arica and F. Yarman-Vural, "An Overview of Character Recognition Focused onOff-line Handwriting”, IEEE Transactions on Systems, Man, and Cybernetics, Part C:Applications and Reviews, 2001, 31(2), pp. 216 - 233. https://doi.org/10.1109/5326.941845.

[24] U. Bhattacharya, and B. B. Chaudhuri, "Handwritten numeral databases of Indianscripts and multistage recognition of mixed numerals," IEEE Transaction on Patternanalysis and machine intelligence, vol.31, No.3, pp.444-457, 2009. https://doi.org/10.1109/TPAMI.2008.88.

[25] U. Pal, T. Wakabayashi and F. Kimura, "Handwritten numeral recognition of sixpopular scripts," Ninth International conference on Document Analysis and RecognitionICDAR 07, Vol.2, pp.749. $753,2007$.

[26] K.Vijayakumar·C,Arun, "Continuous security assessment of cloud based applications using distributed hashing algorithm in SDLC", Cluster Computing DOI 10.1007/s10586-017-1176-x,Sept 2017

[27] K.Vijayakumar.C,Arun, Analysis and selection of risk assessmen frameworks for cloud based enterprise applications", Biomedical Research, ISSN: 0976-1683 (Electronic), January 2017

[28] K. Vijayakumar and C. Arun, "Continuous Security Assessment of Applications in Cloud Environment", International Journal of Control Theory and Applications, ISSN: 0974-5645 volume No. 9(36), Page No. 533-541, 2016. 